\title{
The Chloroplast Import Receptor Toc34 Functions as Preprotein-Regulated GTPase
}

\author{
Marko Jelic ${ }^{1}$, Natalia Sveshnikova ${ }^{2}$, \\ Michael Motzkus², Patric Hörth ${ }^{3}$, Jürgen Soll1 \\ and Enrico Schleiff1,* \\ ${ }^{1}$ Botanisches Institut, LMU München, Menzinger Str 67, \\ D-80638 München, Germany \\ 2 Botanisches Institut der Universität Kiel, D-24118 Kiel, \\ Germany \\ ${ }^{3}$ Institut für Biologie II, Universität Freiburg, \\ D-79104 Freiburg, Germany \\ ${ }^{*}$ Corresponding author
}

Toc34 is a protein of the chloroplast outer envelope membrane that acts as receptor for preproteins containing a transit sequence. The recognition of preproteins by Toc34 is regulated by GTP binding and phosphorylation. The phosphorylation site of Toc34 is located at serine 113, close to the postulated triphosphate binding site. This can explain the down-regulation of Toc34 by phosphorylation, resulting in the loss of GTP binding. Vice versa, GTP but not GDP binding of Toc34 influences the phosphorylation. The nucleotide specificity of Toc34 is not only determined by the classical nucleotide binding domains but by a non-typical region at the $\mathrm{N}$-terminus of the protein. As a result, the GTP binding properties are unusual, since the triphosphate moiety of GTP is bound with higher affinity than the purine base. Purified Toc34 hydrolyses GTP at a low rate, which could regulate the receptor function. The rate of hydrolysis is greatly stimulated by a precursor protein.

Key words: Chloroplast import/GTP binding /

Phosphorylation/Preprotein recognition.

\section{Introduction}

Most of the proteins that reside in chloroplasts are nuclearly encoded gene products. These proteins are translated in the cytoplasmic compartment with an $\mathrm{N}$-terminal cleavable targeting sequence or presequence. Phosphorylation of the presequence by a cytosolic protein kinase leads to the formation of a guidance complex. This complex targets the precursor proteins to the surface of the chloroplast (Schleiff and Soll, 2000). Phosphorylation of the transit sequence also stimulates the recognition of the precursor by the import receptor Toc34 (Translocon at the outer envelope of chloroplasts of $34 \mathrm{kDa}$ ) (Schnell et al., 1997; Sveshnikova et al., 2000; Schleiff et al., 2001). Prior to translocation over the envelope membranes the preprotein has to be dephosphorylated (Waegemann and Soll, 1996). Protein translocation into chloroplasts is then assisted by several proteins in the envelope membranes and in the stroma (Keegstra and Cline, 1999; Schleiff and Soll, 2000). This process was found to be energy dependent (Flügge and Hinz, 1986; Schindler et al., 1987; Olsen et al., 1989; Olsen and Keegstra, 1992; Young et al., 1999). After translocation the signal is cleaved off, resulting in the mature form of the protein or in the precursor form for thylakoid targeting.

Two outer envelope proteins were identified as preprotein receptors, namely Toc34 (Kouranov and Schnell, 1997; Gutensohn et al., 2000; Sveshnikova et al., 2000; Schleiff et al., 2001) and Toc159 (Hirsch et al., 1994; Kessler et al., 1994; Bölter et al., 1998; Bauer et al. , 2000; Hiltbrunner et al., 2001). Both proteins contain a GTP binding side and Toc159 contains an additional ATP binding side (Hirsch et al., 1994; Kessler et al., 1994; Seedorf et al., 1995). They can further be phosphorylated (Sveshnikova et al., 2000) and share a high homology within their GTP binding domain. Toc34 also shows homology with small GTPases, especially with members of the h-Ras21p family (Schleiff et al., 2001).

The mode of preprotein recognition by Toc34 was investigated in some detail (Kouranov and Schnell, 1997; Gutensohn et al., 2000; Sveshnikova et al., 2000; Schleiff et al., 2001). The affinity for preprotein binding by Toc34 is enhanced by transit sequence phosphorylation (Sveshnikova et al., 2000; Schleiff et al., 2001). Furthermore, the binding of stroma targeted precursor of the small subunit of ribulose 1,5 biphosphate carboxylaseoxygenase (Rubisco, preSSU) also requires GTP binding by Toc34, while the affinity is drastically decreased in the presence of GDP (Gutensohn et al., 2000; Sveshnikova et al., 2000; Schleiff et al., 2001). Phosphorylation of Toc34 was found to inhibit GTP binding and hence preprotein recognition (Sveshnikova et al., 2000). Therefore the cycle of preprotein recognition by Toc34 regulates by GTP/GDP exchange and phosphorylation. However, the molecular details remained unknown.

Here we demonstrate that the phosphorylation site of Toc34 is serine 113. Furthermore, we show that GTP but not GDP binding inhibits phosphorylation. Therefore, GTP binding and receptor phosphorylation constitute a dominant switch between the active and inactive state of receptor Toc34. Toc34 recognises the triphosphate moiety of GTP with high affinity, whereas the purine-ring of GTP is not as stable associated since GDP can be replaced by addition of ATP or XTP. Toc34 hydrolyses GTP in a precursor stimulated manner. A model of GTP-medi- 
ated binding and GDP-stimulated dissociation of stromal targeted proteins is presented.

\section{Results}

\section{The Phosphorylation Site of Toc34}

Toc34 can be phosphorylated in situ in isolated envelope membranes upon addition of $[\gamma-32 \mathrm{P}]$-ATP (Sveshnikova et al., 2000). In order to demonstrate that Toc34 is also phosphorylated in vivo, isolated chloroplasts were solubilised immediately after purification and Toc34 was immunoprecipitated. The immunoprecipitated proteins were separated by SDS-PAGE and immunoblotted either with Toc34 antibodies (Figure 1A, lane 2) or with phosphoserine antibodies (Figure 1A, lane 4). The phosphoserine antiserum clearly decorated Toc34 from chloroplasts, demonstrating that Toc34 exists, at least partially, in an phosphorylated form in situ. For a control of the im-

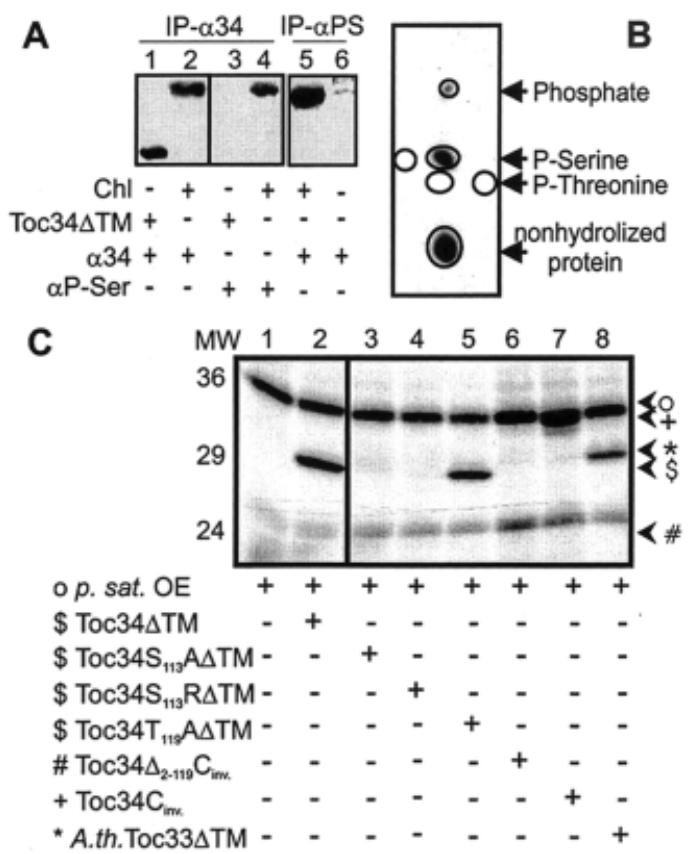

Fig. 1 Analysis of the Phosphorylation Site of Toc34. (A) Toc34 4 TM (lanes 1 and 3) or Toc34 present in chloroplasts (Chl, lanes 2, 4 and 5) was immunoprecipitated by Toc34 (IP$\alpha 34$, lane $1-4$ ) or phosphoserine antibodies (IP- $\alpha P S$, lane 5). The precipitate was immunodecorated with Toc34 ( $\alpha 34$, lanes 1 , 2, 5 and 6) or phosphoserine antibodies ( $\alpha$ P-Ser, lanes 3 and 4). In lane 6, antibodies against phosphoserine were immunodecorated with Toc34 antibodies. (B) Toc34 was phosphorylated using $\left[\gamma^{-32} \mathrm{P}\right]-$ ATP by the kinase present in outer envelopes, hydrolysed and amino acids separated. Ninhydrin-stained phosphoamino acid markers phosphoserine (P-serine) and phosphothreonine (P-threonine) are indicated. (C) Toc34 and mutants were phosphorylated by outer envelope of Pisum sativum. 'o' indicates the endogenous Toc34, ' $\$$ ' the position of Toc34 $\Delta$ TM (lane 2), Toc34S ${ }_{113} \mathrm{~A} \Delta \mathrm{TM}$ (lane 3), Toc34S ${ }_{113} \mathrm{R} 4 \Delta \mathrm{TM}$ (lane 4), Toc34 $T_{119} \mathrm{~A} \Delta \mathrm{TM}$ (lane 5), '\#' the position of Toc34 $\Delta_{2-119} \mathrm{C}_{\text {inv. }}$ (lane 6), '+' the position of Toc34 $\mathrm{C}_{\text {inv. }}$ (lane 7 ) and '*' the position of A.th. Toc33 $\Delta \mathrm{TM}$ (lane 8). munoprecipitation reaction and phosphoserine antibody reactivity, heterologously expressed Toc34 $\Delta \mathrm{TM}$ was immunoprecipitated by Toc34 antiserum. The precipitated polypeptides were not recognised by the phosphoserine antibody, since expression of the protein in E.coli does not result in phosphorylation (Figure 1A, lanes 1 and 3). When phosphoserine antibodies were used to immunoprecipitate phosphorylated outer envelope proteins from isolated chloroplasts, Toc34 could again be identified (Figure 1A, lane 5). From this we conclude that Toc34 is phosphorylated in situ and most likely also in vivo. To clarify if Toc34 is phosphorylated solely on a serine residue, Toc34 $\Delta \mathrm{TM}$ was phosphorylated using outer envelope membranes in the presence of $\left[\gamma^{32} \mathrm{P}\right]-\mathrm{ATP}$, re-purified by affinity chromatography, hydrolysed and phosphoamino acids were determined. Phosphoserine was found as the only labelled amino acid (Figure 1B). To determine the phosphorylation site, Toc $34 \Delta \mathrm{TM}$ was phosphorylated by the kinase present in the outer envelope followed by trypsin or Glu-C peptidase treatment. In addition, Toc34 present in the outer envelope was phosphorylated and proteolytically digested as well. Fragments were subjected to a reversed-phase chromatography and fractions were tested for radioactivity. We observed for both proteins an almost identical fragmentation and phosphorylation pattern (data not shown). In order to identify the phosphorylation site we used two approaches. The protein was phosphorylated by unlabelled ATP followed by proteolytic cleavage. Peptide fractions were collected and pooled based on radioactive pattern obtained before and subjected to mass spectrometry. Furthermore, Toc34 $\Delta \mathrm{TM}$ phosphorylated by outer envelope membranes was treated with chymotrypsin and all peptides were subjected to mass spectrometry without further purification in order to analyse the homogeneity of the phosphorylation. Both approaches resulted in the detection of a single phosphorylation site at serine 113 .

To confirm this observation we created several point mutations of Toc34 replacing serine at position 113 by an alanine or by an arginine. In addition, threonine 119 was replaced by an alanine. The phosphorylation of Toc34 and the different mutants revealed that the replacement of serine 113 resulted in a loss of phosphorylation (Figure $1 \mathrm{C}$, lane $2 \mathrm{vs}$. lanes 3 and 4 ). The replacement of threonine did not result in a reduction of phosphorylation (Figure 1C, lane 2 vs. lane 5). Toc34 containing an inverted C-terminus (May and Soll, 1998) was phosphorylated (Figure 1C, lane 7, slightly smaller than endogenous Toc34). The same protein lacking the amino acids $2-119$ was not phosphorylated, corroborating our finding that the phosphorylation site is located in this N-proximal region. We always observed low but constant phosphorylation of a polypeptide of about $24 \mathrm{kDa}$. This polypeptide did not coimmunoprecipitate with Toc34 antibodies (data not shown) and thus does not represent a proteolytic fragment of Toc34. The exact nature of the $24 \mathrm{kDa}$ protein remains to be determined. Nevertheless, the results allow 
us to conclude that Toc34 from pea is phosphorylated at amino acid position 113.

In order to test whether phosphorylation of Toc34 occurs also in other plants, the Toc34 homologue form Arabidopsis thaliana, Toc33, was used in the phosphorylation assay. As can be seen in Figure $1 \mathrm{C}$, lane 8 , also this protein becomes phosphorylated. Therefore, phosphory-

A
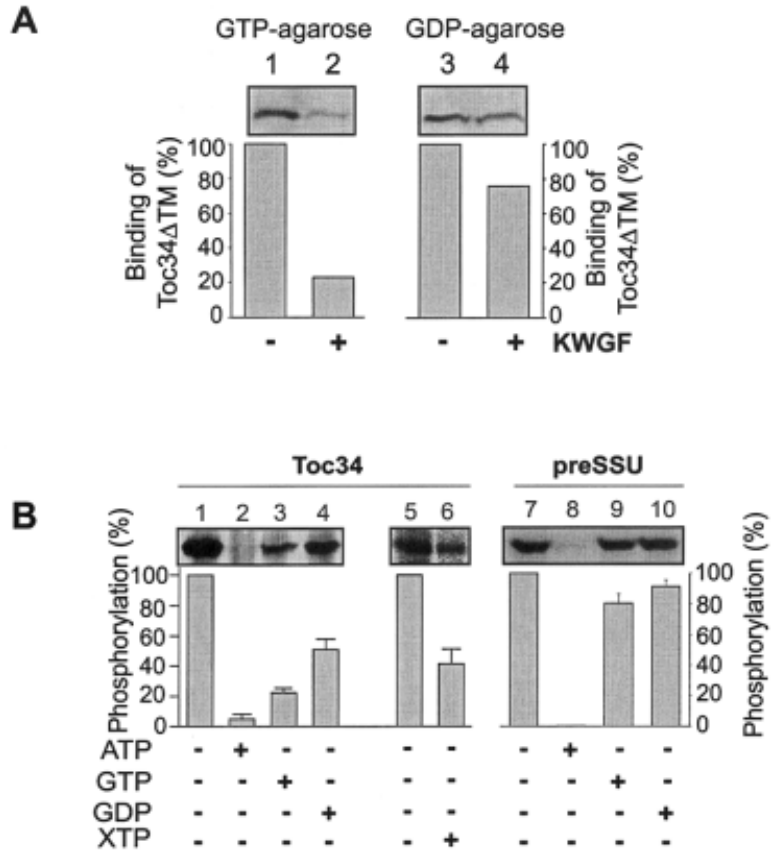

C

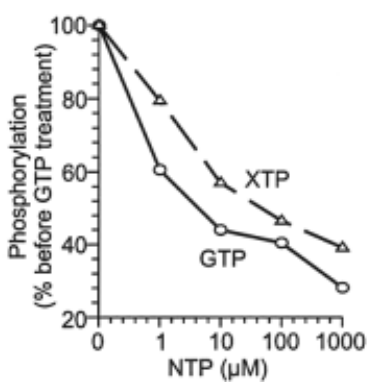

Fig. 2 Phosphorylation Inhibits GTP Binding and vice versa. (A) Toc34 $\Delta$ TM before (lanes 1 and 3) and after phosphorylation using a kinase containing wheat germ fraction (KWGF, lanes 2 and 4) was incubated with GTP (lanes 1 and 2) or GDP agarose (lanes 3 and 4). Shown is the amount of Toc34 $\Delta$ TM remaining on the nucleotide agarose. The binding of Toc $34 \Delta \mathrm{TM}$ was quantified and compared to the binding before phosphorylation (histogram). (B) Toc34 $4 \mathrm{TM}$ (lanes 1-6) or preSSU (lanes 7 - 10) were phosphorylated by a kinase containing wheat germ fraction using $\left[\gamma^{-32}\right.$ P]-ATP before (lanes 1, 5 and 7 ) and after preincubation with $1 \mathrm{~mm}$ ATP (lanes 2 and 8), $1 \mathrm{~mm}$ GTP (lanes 3 and 9), $1 \mathrm{~mm}$ GDP (lanes 4 and 10) or $1 \mathrm{~mm}$ XTP (lane 6). The phosphorylation was quantified and compared to the phosphorylation in the absence of nucleotides (histogram). (C) The outer envelope was incubated with increasing amounts of GTP- $\gamma$-S (open circle) or XTP (open triangle) followed by phosphorylation using [ $\left.\gamma^{-32} \mathrm{P}\right]-$ ATP. The envelope proteins were then separated on SDS-Page and phosphorylation of Toc34 was quantified as described in Materials and Methods. Values are presented as percent of the phosphorylation achieved without GTP or XTP treatment. lation of the Toc34/33 receptor proteins seems to be a general regulatory step during chloroplast protein translocation.

\section{Interplay between GTP Binding and Phosphorylation}

In order to understand the interplay of Toc34 phosphorylation and nucleotide binding we investigated the effect of GTP and GDP binding on phosphorylation as well as the effect of phosphorylation on GTP and GDP binding. When Toc34 $\Delta$ TM was phosphorylated, binding to a GTP affinity matrix was reduced by $80 \%$ (Figure $2 \mathrm{~A}$, lanes 1 and 2), whereas binding to the GDP affinity matrix was only reduced by 20\% (Figure 2A, lanes 3 and 4). However, when Toc34 was incubated with agarose no interaction could be observed before or after phosphorylation (data not shown). Earlier work has established that about $75 \%$ of Toc34 is phosphorylated (Sveshnikova et al., 2000). This suggests that phosphorylation could occur also in the GDP-bound state of Toc34 and not only in the nucleotidefree state of Toc34, since phosphorylated Toc34 still binds to GDP albeit with lower affinity than the non-phosphorylated one. To test this idea we investigated the influence of nucleotide binding of Toc34 on phosphorylation directly. Toc34 $\Delta$ TM was preincubated with ATP, GTP, GDP or XTP and subsequently phosphorylated by a protein kinase partially purified from wheat germ extract. A decrease of phosphorylation of Toc34 $\Delta \mathrm{TM}$ was observed in the presence of ATP (Figure 2B, lanes 1 and 2) due to the competition between radioactively labelled ATP and unlabelled ATP, and in the presence of GTP or XTP (Figure 2B, lanes 1 and 3 , lanes 5 and 6), but much less in the presence of GDP (Figure 2B, lanes 1 and 4). In comparison, phosphorylation of preSSU, a protein not interacting with nucleotides, was completely inhibited in the presence of ATP (Figure 2B, lane 8), whereas addition of GTP or GDP did not alter the phosphorylation efficiency of the kinase fraction (Figure 2B, lanes 7, 9 and 10). We therefore conclude that the triphosphate binding of Toc34 inhibits phosphorylation of the receptor (see Discussion). To support this finding we pre-incubated outer envelope membranes with different concentrations of GTP- $\gamma$-S or XTP and initiated phosphorylation by addition of radioactively labelled ATP (Figure 2C). As seen before, phosphorylation of Toc34 was significantly reduced when GTP was present. In the presence of $1 \mathrm{~mm}$ GTP- $\gamma$-S only $28 \%$ and in the presence of $1 \mathrm{~mm}$ XTP only $39 \%$ of Toc34 became phosphorylated in comparison to the control.

\section{Nucleotide Binding Properties of Toc34}

Recognition and release of the targeting sequence by Toc34 is regulated by GTP versus GDP binding (Sveshnikova et al., 2000; Schleiff et al., 2001). In order to determine the affinity difference of Toc34 for various nucleotides, Toc34 $\Delta$ TM was incubated with GTP agarose (Figure 3A, lane 1) or GDP-agarose (Figure 3A, lane 8). Toc34 $\Delta$ TM agarose was washed with increasing concentrations of GDP, GTP or ATP. The remaining Toc34 $\Delta$ TM 


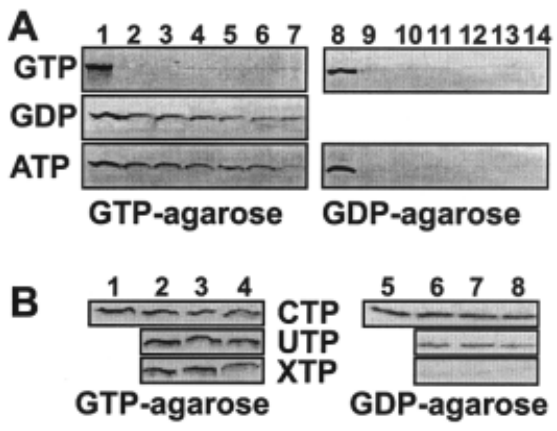

Fig. 3 Toc34 $\Delta \mathrm{TM}$ Interaction with Nucleotides Is Initiated by the Triphosphate.

(A) Toc34 $\Delta$ TM was preincubated with GTP agarose (lanes 1-7) or GDP agarose (lanes 8-14). Shown is the amount of Toc34 $\Delta$ TM remaining on the nucleotide agarose without competition (lanes 1 and 8) and after addition of $0.1 \mu \mathrm{M}$ (lanes 2 and 9), $1 \mu \mathrm{m}$ (lanes 3 and 10), $10 \mu \mathrm{m}$ (lanes 4 and 11), $100 \mu \mathrm{m}$ (lanes 5 and 12) $1 \mathrm{~mm}$ (lanes 6 and 13) and $10 \mathrm{~mm}$ (lanes 7 and 14) of the indicated nucleotide GTP, GDP or ATP. (B) The same experiment was performed using CTP, UTP and XTP for competition. Shown is Toc34 $\Delta \mathrm{TM}$ remaining on the nucleotide agarose without competition (lanes 1 and 5) and after addition of $0.1 \mu \mathrm{M}$ (lanes 2 and 6), $10 \mu \mathrm{m}$ (lanes 3 and 7) and $1 \mathrm{~mm}$ (lanes 4 and 8 ) of the indicated nucleotide.
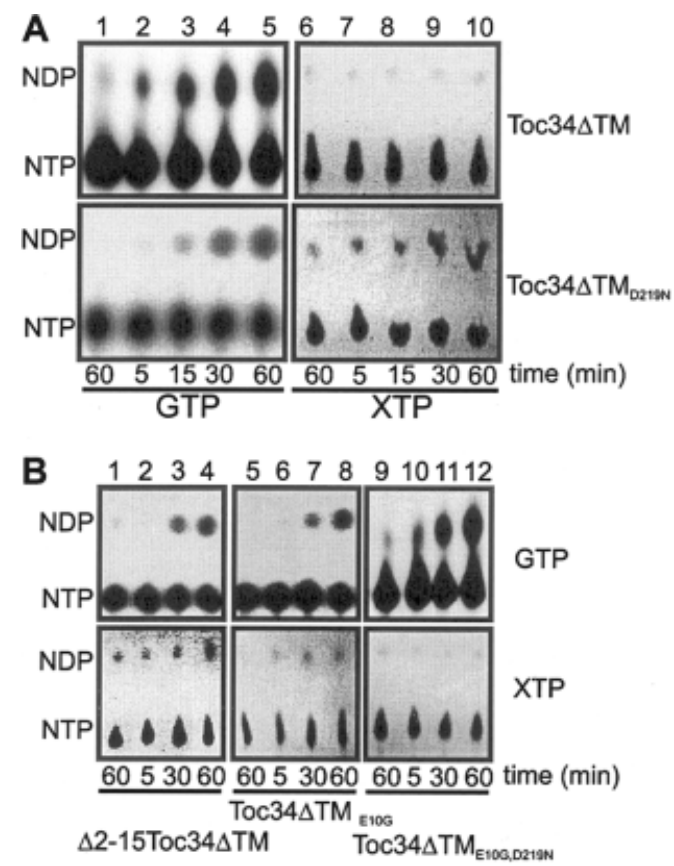

Fig. 4 Toc34 $\Delta$ TM Hydrolysis of GTP.

(A) Toc34 $\Delta \mathrm{TM}$ or Toc34 $\Delta \mathrm{TM}_{\mathrm{D219N}}$ (100 ng) was incubated for 5 , 15,30 and 60 min with $1 \mu \mathrm{Ci}\left[\alpha_{-}{ }^{32} \mathrm{P}\right]-\mathrm{GTP}$ (lanes 2, 3, 4 and 5) or 2 mM XTP (lanes 7, 8, 9 and 10). Self hydrolysis of GTP (lane 1) or XTP (lane 6) after 60 min under experimental conditions is shown. (B) $\Delta 2-15 T$ Toc34 $\Delta T M$ (lanes 2, 3 and 4), Toc34 $\Delta T_{\mathrm{E}_{10 G}}$ (lanes 6, 7, and 8) or Toc34 $\Delta \mathrm{TM}_{\mathrm{E} 10 \mathrm{G}, \mathrm{D} 219 \mathrm{~N}}$ (lanes 10, 11 and 12) $(100 \mathrm{ng})$ were incubated for 5,30 and $60 \mathrm{~min}$ with $1 \mu \mathrm{Ci}[\alpha-32 \mathrm{P}]-$ GTP (upper panel) or 2 mM XTP (lower panel). Self hydrolysis of GTP or XTP after 60 min under the experimental conditions used is shown in lanes 1, 5 and 9. was eluted from the matrix by SDS-containing buffer and analysed by immunoblotting. Interestingly, Toc34 $\Delta T M$ bound to GTP-agarose eluted only partially even after treatment with $1 \mathrm{~mm}$ ATP or $1 \mathrm{~mm}$ GDP, but completely after treatment with GTP (Figure 3A, left panel). In contrast, a low concentration of ATP or GTP was sufficient to remove Toc34 $\Delta$ TM almost completely from GDP-agarose (Figure 3A, right panel).

To investigate whether Toc34 $\Delta \mathrm{TM}$ recognises all nucleoside triphosphates or only those containing purine ring systems we used CTP, UTP and XTP to elute Toc34 $\triangle$ TM from the GTP or GDP containing matrix (Figure $3 \mathrm{~B}$ ). CTP and UTP did not disrupt the interaction of Toc34 $\Delta$ TM with GTP (Figure 3B, left panel). Only in the presence of $1 \mathrm{mM}$ XTP the amount of bound Toc34 $\Delta$ TM seemed slightly reduced. In contrast to elution of Toc34 $\triangle T M$ from the GDP matrix by ATP and GTP (Figure $3 A$ ), CTP and UTP did not influence the binding of Toc34 $\triangle T M$ to the GDP matrix, whereas XTP resulted in an elution similar to ATP and GTP (Figure 1B, right panel). These findings suggest that Toc $34 \Delta \mathrm{TM}$ binds the triphosphate form of nucleosides containing a purine ring with higher affinity than GDP.

\section{Intrinsic Regulation of GTP Hydrolysis by Toc34}

In order to establish GTP hydrolysis by Toc34 an in vitro assay using purified Toc34 $\Delta \mathrm{TM}$ was developed. Purified Toc34 $\Delta$ TM hydrolyses GTP at a slow rate (Figure 4A, Toc34 $\Delta T M$, lanes 2-5). Although Toc34 binds to XTP (Figure 3), we did not observe hydrolysis of XTP (Figure $4 \mathrm{~A}$, Toc34 $\Delta \mathrm{TM}$, lanes $7-10)$. We then replaced aspartic acid at position 219 by asparagine, a mutation within the conserved nucleotide binding domain that was found to alter the GTP specificity to a XTP specificity (Hwang and Miller, 1987; Schmidt et al., 1996). Toc34 bearing this mutation was now able to hydrolyse XTP (Figure 4A, Toc34 $\Delta \mathrm{TM}_{\mathrm{D} 219 \mathrm{~N}}$, lanes $7-10$ ), while GTP hydrolysis was largely suppressed (Figure $4 \mathrm{~B}$, Toc $34 \Delta \mathrm{TM}_{\mathrm{D} 219 \mathrm{~N}}$, lanes $2-5)$. We conclude that Toc34 is a GTPase and that the GTPase activity measured is not due to a protein that copurifies with Toc34. Besides the classical aspartic acid point mutation we identified a mutation in a region not present in other small GTPases, but which influences the GTPase activity of Toc34. When the first fifteen amino acids of Toc34 were deleted, the specificity of Toc34 for GTP was lost and $\Delta 2-15$ Toc34 $\Delta$ TM was able to hydrolyse XTP (Figure 4B, lanes 2-4). A similar but not as pronounced effect was observed when glutamic acid at

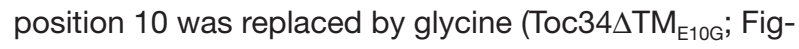
ure $4 \mathrm{~B}$, lanes $6-8$ ). The XTP hydrolysing activity of both mutants was lower than that found for Toc34 $\Delta \mathrm{TM}_{\mathrm{D} 219 \mathrm{~N}}$. The double mutant Toc34 $\Delta \mathrm{TM}_{\mathrm{E} 10 \mathrm{G}, \mathrm{D} 219 \mathrm{~N}}$ was able to hydrolyse GTP but not XTP (Figure 4B, lanes 10-12). Therefore, we conclude that Toc34 contains not only the known motifs conserved in small GTPases but also a novel region determining nucleotide selectivity at its extreme N-terminus. 
To further characterise the GTP hydrolysis, mutants of Toc34 were tested that contained mutations at the phosphorylation side. Interestingly, the serine to alanine replacement also resulted in a loss of hydrolysis (Figure 5A, lanes 4 and 10), whereas the serine to arginine exchange resulted in a magnesium insensitivity (Figure 5A, lanes 3 and 9). The threonine replacement at position $119 \mathrm{had}$ no influence on GTP hydrolysis (Figure 5A, lanes 5 and 11). We conclude that the status of the phosphorylation site influences the hydrolytic activity of Toc34. Furthermore, the homologue from Arabidopsis thaliana, atToc33, hydrolysed GTP in a magnesium-dependent manner (Figure $5 \mathrm{~A}$, lanes 2 and 8 ), underlining that this polypeptide acts in a similar manner.

In order to determine the nucleotide specificity of Toc34 GTPase activity, hydrolysis assays were carried out in the absence or presence of XTP, UTP, ATP or GDP. In line with the results shown in Figure 3, only XTP was able to compete for GTP binding and subsequently for hydrolysis by Toc34 $\Delta \mathrm{TM}$ (Figure 5B). In line with the observation that ATP and GDP could replace GTP only at high concentrations (Figure $3 \mathrm{~A}$ ), the hydrolysis of GTP by Toc34 $\Delta$ TM could not be competed for by addition of ATP or GDP (Figure 5C). Only at a thousand-fold excess of ATP or GDP a slight reduction of GTP hydrolysis was observed (Figure $5 \mathrm{C}$ ). However, this reduction was also
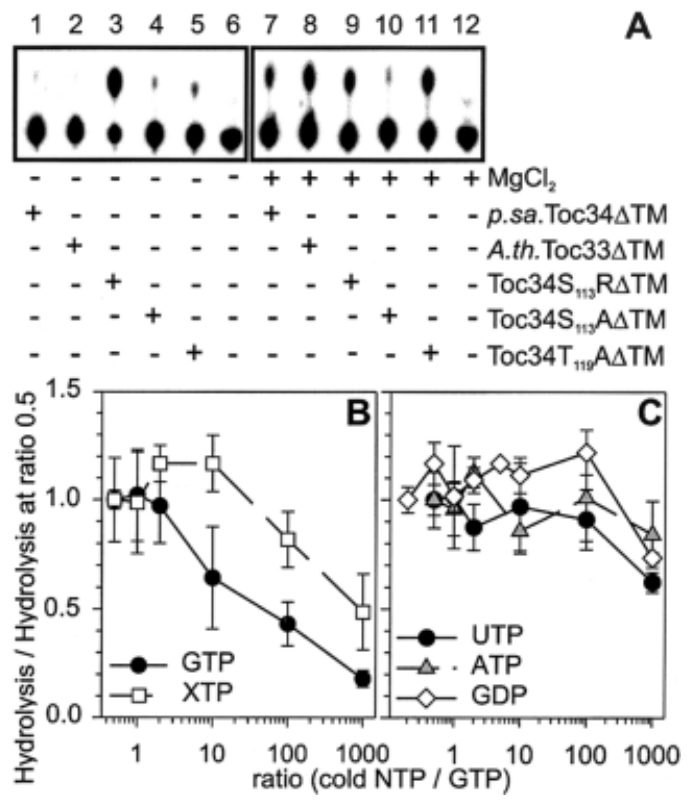

Fig. 5 Properties of GTP Hydrolyses by Toc34 $\Delta$ TM.

(A) One-hundred ng of p.sa. Toc34 $3 \mathrm{TM}$ (lanes 1 and 7), a.th. Toc33 $\Delta$ TM (lanes 2 and 8), Toc34S ${ }_{113} R \Delta T M$ (lanes 3 and 9),

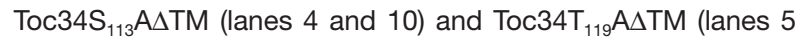
and 11) were incubated with $35 \mathrm{nM}\left[\alpha-{ }^{32} \mathrm{P}\right]-\mathrm{GTP}$ in the absence (lanes 1-6) or presence of $\mathrm{MgCl}_{2}$ (lanes 7-12) for $30 \mathrm{~min}$ and hydrolysis is shown. $(B, C)$ Hydrolysis of $35 \mathrm{nM}\left[\alpha^{-32} \mathrm{P}\right]-\mathrm{GTP}$ by $0.1 \mu \mathrm{g}$ Toc34 $3 \mathrm{TM}$ was determined after $30 \mathrm{~min}$ in the presence of increasing amounts of XTP (B, open square), GTP (B, closed circle), GDP (C, open diamond), UTP (C, closed circle) and ATP (C, grey triangle). The amount of hydrolysed NTP was compared to the hydrolysis in the presence of 0.1 -fold amount of competitor. observed for UTP, which was not found to release Toc34 3 TM from the GTP or GDP affinity matrix. This is consistent with the hypothesis that Toc34 recognises the triphosphate with higher affinity than the diphosphate of guanosine, as suggested before. It further strengthens the idea that Toc34 cannot only recognise the guanosine ring but to some extent the xantosine ring.

\section{Analysis of the GTPase Activity of Toc34}

A detailed analysis of the hydrolysis of GTP by Toc34 $\Delta$ TM252 (Figure $6 \mathrm{~A}$ ) revealed a very slow maximal

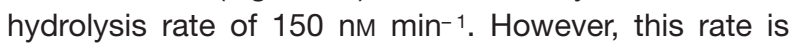
comparable to the intrinsic GTP hydrolysis rate of other small GTPases (for example, see Downward et al., 1990; Zhang et al., 1997; Albert et al., 1999). The catalytic constant of $0.02 \mathrm{~min}^{-1}$ is also comparable to the intrinsic catalytic constant found for other GTPases (for example see Ruetthard et al., 2001). The analysis further revealed a Michaelis-Menten constant of $20 \mu \mathrm{M}$ (Figure 6A, inset). We conclude that Toc34 can act as a GTPase in vivo, but that its rate of hydrolysis is most likely stimulated by proteinaceous effectors.

Therefore, we tested whether such stimulation can be observed in the presence of precursor protein. preSSU and preOE33, but not the mature form of SSU, clearly

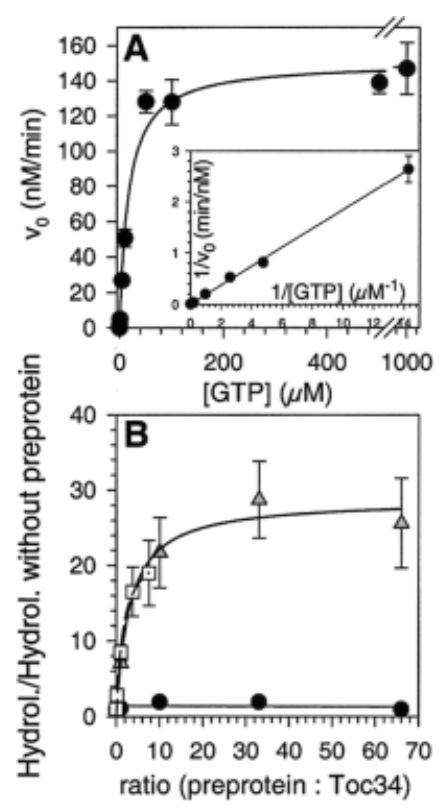

Fig. 6 GTPase Activity of Toc34 4 TM Is Stimulated by Preproteins.

(A) Michaelis-Menten kinetics of the hydrolysis were determined using $5 \mu \mathrm{g}$ of protein. For this, the initial rate constant $v_{\mathrm{o}}$ of the hydrolysis of different amounts of GTP was determined. The insert shows the inverted plot of the Michaelis-Menten kinetics for $K_{\mathrm{m}}$ determination. (B) Toc34 $3 \mathrm{TM}$ (100 ng) was incubated for 30 min with $\left[\alpha-{ }^{32} \mathrm{P}\right]$-GTP in the presence of increasing amounts of mSSU (closed circle), preSSU (open square) and preOE33 (grey triangle). The GTP hydrolysis was compared to the initial hydrolysis of Toc34 $\Delta \mathrm{TM}$ in the absence of a preprotein. For panels, each data point reflects the average of at least 4 independent experiments. 
stimulated hydrolysis of GTP up to 28-fold (Figure 6B). The half-maximal of stimulation was reached at a 2-fold molar excess of the preprotein over Toc34, again documenting the high affinity of Toc34 $\Delta \mathrm{TM}$ for the preprotein in the presence of GTP. Therefore, association of Toc34 with the preprotein is one regulatory mechanism of the GTPase activity of Toc34 and its receptor function.

\section{Discussion}

\section{Phosphorylation of Toc34}

Phosphorylation/dephosphorylation cycles are powerful tools to regulate diverse processes, such as biochemical pathways or cell and organelle cycle (Hardie, 1999). Specifically, protein phosphorylation has been demonstrated to influence solute transport as well as protein translocation (Gruss and Dobberstein, 1999; Deltrot et al., 2000). Toc34, a GTP-dependent protein import receptor represents a prominent phosphoprotein of the chloroplast outer envelope (Figure 1). The activity of Toc34 can be regulated by phosphorylation at serine 113 (Figure 1) causing the inactivation of its GTP binding capacity (Figure 2) and therefore resulting in a desensitisation. Similar effects have been demonstrated for the eukaryotic initiation factor (Pain, 1996) or dynamine (Robinson et al., 1993). Our data demonstrate that the phosphorylation shuts off the preprotein recognition (Sveshnikova et al., 2000) and therefore regulates translocation via the Toc34 receptor. The cell might require such regulatory mechanism to adopt the protein repertoire of the chloroplast to the actual biochemical needs, e.g. under high light conditions, which require a response of the photosynthetic machinery but also of proteins involved e.g. in photorespiration. Recently it could be demonstrated that the import rates of preSSU at different stages of the plastid development are drastically altered (Su et al., 2001). It was further demonstrated that this is due to a reduction of the preprotein recognition sites, which can also be the result of an increase of phosphorylated receptor proteins. These finding could also indicate a development-dependent phosphorylation of Toc34 as a general regulatory mechanism. Alterations of illumination conditions result in changes of the redox state of the chloroplast. The Tic-complex contains Tic55, a Rieske-type iron sulphur protein, which could be a component of a redox sensing circuit at the inner envelope (Caliebe et al., 1997). The Toc complex, as exemplified here for Toc34, is regulated by phosphorylation and nucleotide binding. In combination such a system might build a regulatory network between the organelle and the parent cell (nucleus) to control protein targeting and organelle function.

\section{Toc34, a GTPase with Unique Properties}

Typical small GTPases have five conserved regions (G1G5; Bourne et al., 1991). They specifically interact with
GTP, but their intrinsic hydrolysis rate is rather low (Downward et al., 1990; Zhang et al., 1997; Albert et al., 1999). Toc34 recognises all nucleoside triphosphates containing a purine ring, but GTP with highest affinity (Figure 3). This is consistent with earlier observations that ATP cannot compete for GTP binding (Kessler et al., 1994; Sveshnikova et al., 2000). Hydrolysis was only observed for GTP (Figure 4). GTP was hydrolysed by Toc34 with maximal hydrolysis rate (150 nM min-1) which is comparable to the intrinsic hydrolysis rate of other small GTPases (Downward et al., 1990; Zhang et al., 1997; Albert et al., 1999) and already higher than an earlier reported hydrolysis rate for the dimerised form of Toc34 (Sun et al., 2002). The increase of the hydrolysis rate compared to the earlier rate can be explained by the different experimental conditions. Furthermore, the catalytic constant of $0.02 \mathrm{~min}^{-1}$ determined here is similar to the intrinsic activity of other GTPases (Bourne et al., 1991). We therefore conclude that Toc34 acts as GTPase and hydrolysis can only be inhibited by XTP, a nucleotide that does not exist as a free substrate in vivo (Figures 4 and 5).

Mutations within or close to the G5 domain result in conversion of the GTPase to a XTPase (Hwang and Miller, 1987; Schmidt et al., 1996). Therefore we changed aspartic acid 219 to asparagine (Figure 4). However, analysis of the structure of Toc34 (Sun et al., 2002) revealed that D219 is not part of the G5 domain explaining the basal GTP hydrolysis of the D219N mutant. However, Toc34 has properties that are not common for GTPases: the extreme $\mathrm{N}$-terminal region regulates the GTPase specificity and activity and the state of the phosphorylatable serine 113 influences the hydrolysis by regulating GTP binding. The first observation can be explained by a stabilisation of the G2 and G5 structure by the N-terminus. Analysis of the structure of Toc34 (Sun et al., 2002) revealed that glycine 13 is in close proximity to isoleucine 56 and alanine 18 to serine 220. Destabilisation of this region by deletion of the $\mathrm{N}$-terminus or alteration of the amino acid composition would therefore result in loss of the conformational stability and in the observed loss of specificity. Furthermore, the phosphorylation site is located in the switch II domain (Sun et al., 2002) explaining the loss of hydrolysis. In addition, the close proximity of serine 113 to arginine 76 located within the same loop as the magnesium-bound glutamic acid 73 (Sun et al., 2002) explains the observed desensibilisation of hydrolysis to magnesium when serine was replaced by arginine (Figure 5). Additionally, the close proximity of the large phosphate group after phosphorylation to the negatively charged arginine will cause a deformation of the magnesium binding loop by electrostatic interaction, explaining the influence of phosphorylation on GTP binding and subsequent hydrolysis.

Most GTPases are regulated by very specific guanine nucleotide exchange factors (GEF; Cherfils and Chardin, 1999) and GTPase activating proteins (GAP; Scheffzek et al., 1998). Recently it was suggested that dimerisation of Toc34 might cause a GTPase activation and therefore 
function as a GAP (Sun et al., 2002). However, the hydrolysis rate of the dimerised form of Toc34 (Sun et al., 2002) was even lower than the rate for Toc34 in the absence of a precursor reported here. In the presence of a transit peptide-containing protein the hydrolysis of GTP was stimulated about 30-fold (Figure 6). Furthermore, the extent of stimulation by preSSU and preOE33 is similar to GAP proteins, which was found to be in the order of two to five orders of magnitude (Albert et al., 1999). We conclude that precursor proteins have a very pronounced effect as GTPase activating proteins in Toc34 function or cooperate in an unknown manner with typical GTPase activating proteins. However, the association of Toc34 with the preprotein gives an additional stimuli to the hydrolysis of GTP by the GTPase. The resulting GDP-Toc34 precursor complex has a lower affinity for the preprotein than the GTP-bound form (4) and allows the preprotein to dissociate from the receptor and continue its passage through the translocon.

\section{Materials and Methods}

\section{General}

$\left[\gamma_{-32}{ }^{32}\right]-A T P(3000 \mathrm{Ci} / \mathrm{mmol})$ and $[\alpha-32 \mathrm{P}]-\mathrm{GTP}(3000 \mathrm{Ci} / \mathrm{mmol})$ were purchased from Amersham Pharmacia Biotech (Freiburg, Germany). All other chemical used were obtained from Roth (Karlsruhe, Germany) or Sigma (Munich, Germany).

Standard procedures like the purification of outer envelopes of chloroplasts or expression are described elsewhere (Seedorf et al., 1995; Sveshnikova et al., 2000). A protein kinase containing fraction (KWGF) was partial purified from wheat germ as described earlier (Waegemann and Soll, 1996).

\section{Introduction of Point Mutations into Toc34 and Protein Expression}

Point mutations were introduced by standard polymerase chain reaction using the previously described Toc34 $\Delta \mathrm{TM}$ construct (Sveshnikova et al., 2000) as template. Point mutations were confirmed by sequencing and constructs expressed and purified as previously described (Sveshnikova et al., 2000). After purification Toc34 $3 \mathrm{TM}$ and mutants were dialysed into the buffer used for the experiments.

\section{Immobilisation of Toc34 onto GTP- or GDP-Crosslinked Agarose}

$0.1 \mu \mathrm{M}$ of purified Toc34 $\Delta \mathrm{TM}$ in buffer A (20 mM Tris-HCl, pH7.5, $120 \mathrm{~mm} \mathrm{NaCl}, 5 \mathrm{~mm} \mathrm{MgCl}$ ) were incubated with GTP or GDP agarose (1 $\mathrm{mm}$ immobilised nucleotide final) for $20 \mathrm{~min}$ at room temperature. After incubation the agarose was washed twice with buffer $A$ and then incubated for $5 \mathrm{~min}$ with different nucleotides as described in the legend to Figure 4. The agarose was then washed three times with buffer $A$ and remaining Toc34 $\Delta$ TM was eluted by adding SDS-sample buffer and boiling at $95^{\circ} \mathrm{C}$ for $3 \mathrm{~min}$. The specificity of the binding was verified by addition of equal amounts of albumin, which did not bind to the column under the conditions used.

\section{GTP and XTP Hydrolysis Assay}

Indicated amounts of Toc34 $4 \mathrm{TM}$ were incubated in $10 \mu \mathrm{l}$ containing $20 \mathrm{~mm}$ Tricine/KOH, $\mathrm{pH} 7.6,1 \mathrm{~mm} \mathrm{MgCl}, 50 \mathrm{~mm} \mathrm{NaCl}$,
$1 \mathrm{~mm}$ DTT with $\left[\alpha^{-32} \mathrm{P}\right]-$ GTP or unlabelled XTP. Nucleotides were separated on PEI-cellulose plates (Merck, Darmstadt, Germany) using $600 \mathrm{~mm} \mathrm{NaH}_{2} \mathrm{PO}_{4}$ at $\mathrm{pH} 3.4$ as running buffer. The hydrolysis of GTP was analysed by the Michaelis-Menten equation; $v_{\mathrm{o}}=V_{\max }[\mathrm{GTP}] /\left([\mathrm{GTP}]+K_{\mathrm{m}}\right)$ were $v_{\mathrm{o}}$ is the initial hydrolysis rate at a given GTP concentration, $V_{\max }$ the maximal rate of hydrolysis and $K_{\mathrm{m}}$ the Michaelis-Menten constant. The catalytic constant $k_{\text {kat }}$ was calculated by; $k_{\text {kat }}=V_{\text {max }} /[\operatorname{Toc} 34 \Delta \mathrm{TM}]_{\mathrm{T}}$.

\section{Treatment of Outer Envelopes with Non-Hydrolysable GTP Analogue}

Outer envelopes were incubated for 10 min with $1 \mathrm{~mm}$ of GTP- $\gamma$ $\mathrm{S}$ in $100 \mu \mathrm{l}$ of buffer $\mathrm{A}$ followed by centrifugation. The envelope was resuspended in buffer $A$ without NTP, again centrifuged and resuspended in phosphorylation buffer.

\section{Phosphorylation of Toc34}

Toc34 $\triangle \mathrm{TM}$ was incubated for 10 min with KWGF and $10 \mathrm{nM}$ or $50 \mathrm{~nm}\left[\gamma^{-32} \mathrm{P}\right]-$ ATP $(3000 \mathrm{Ci} / \mathrm{mmol})$ or $10 \mu \mathrm{M}$ ATP in $20 \mathrm{~mm}$ Tricine/KOH, pH 7.6, $120 \mathrm{~mm} \mathrm{NaCl}, 5 \mathrm{~mm} \mathrm{MgCl}, 0.5 \mathrm{~mm} \mathrm{MnCl}_{2}$ at room temperature. Purified outer envelopes in $20 \mathrm{~mm}$ Tricine/ $\mathrm{KOH}, \mathrm{pH}$ 7.6, $120 \mathrm{~mm} \mathrm{NaCl}, 5 \mathrm{mM} \mathrm{MgCl}, 0.5 \mathrm{mM} \mathrm{MnCl}_{2}$ were incubated for $10 \mathrm{~min}$ at room temperature in $50 \mu \mathrm{l}$ final volume with $10 \mathrm{~nm}\left[\gamma^{-32} \mathrm{P}\right]-$ ATP. Proteins were subjected to SDSPAGE without further treatment and phosphorylation visualised by autoradiography.

\section{Immunoprecipitation of Toc34 Using Toc34 or Anti-Phosphoserine Antibodies}

Protein A-Sepharose was preloaded with antisera to Toc34 or phosphoserine, respectively. Chloroplasts were solubilised using $1 \%$ SDS followed by a 10 -fold dilution into $20 \mathrm{~mm}$ Tris $\mathrm{pH} 7.5$, $120 \mathrm{~mm} \mathrm{NaCl}$, and 1\% NP-40. Purified Toc34 was treated in the same manner. The solubilised chloroplasts were centrifuged for $5 \mathrm{~min}$ at $15000 \mathrm{~g}$ and supernatant incubated with the affinity matrix for 2 hours at $22^{\circ} \mathrm{C}$. Then, the matrix was washed several times with the indicated buffer and eluted by addition of SDSsample buffer and boiling at $95^{\circ} \mathrm{C}$ for $3 \mathrm{~min}$. The proteins were separated on SDS-PAGE and immunodecorated as indicated.

\section{Phosphoamino Acid Analysis of Toc34}

Phosphoamino acid analysis was performed as earlier described (Soll et al., 1988). In brief, phosphorylated Toc34 $\Delta$ TM was hydrolysed by $6 \mathrm{~N} \mathrm{HCl}$ in presence of unlabeled phosphoamino acids (phosphotyrosine, phosphothreonine, phosphoserine, $20 \mu \mathrm{g}$ each). The solvent was evaporated and the residual resuspended in water. The solution was spotted onto a precoated Silica thin-layer plate (Merck, Kieselgel 60) and electrophoresed using glacial acetic acid/formic acid/ $/ \mathrm{H}_{2} \mathrm{O}(78 / 25 / 897)$. The phosphoamino acids were located by ninhydrin staining and radioactivity was detected by autoradiography.

\section{Isolation of Phosphorylated Peptides of Toc34}

Phosphorylated Toc34 (overexpressed or wtToc34 from outer envelopes) was excised from the gel by electro-elution using an electro-eluter (C.B.S. Scientific company, Del Mare, USA) following the guidelines of the manufacturer. The eluted solution was precipitated by trichloracetic acid and the pellet resuspended in $50 \mathrm{~mm} \mathrm{NH}_{4} \mathrm{KCO}_{3}$. Twenty $\mu \mathrm{g}$ of resuspended Toc34 was digested using $10 \mu \mathrm{g}$ trypsin or chymotrypsin at $37^{\circ} \mathrm{C}$. After $180 \mathrm{~min}$ a further $10 \mathrm{\mu g}$ were added and digestion continued at the same conditions for $180 \mathrm{~min}$. The protein was then lyophilised and resuspended in $0.1 \%$ TFA. Digestion products 
were separated on a reverse-phase column ultrasphere ODS $5 \mu$ (Beckmann) using a $60 \mathrm{ml}$ gradient from 15\% acetonitrile/0.1\% TFA to $55 \%$ acetonitrile/ $0.1 \%$ TFA, a flow rate of $1 \mathrm{ml} / \mathrm{min}$ and a fraction size of $0.5 \mathrm{ml}$. Fractions were then lyophilised for further treatment.

\section{Mass Spectrometry}

Mass spectra were obtained on Finnigan MAT TSQ700 tandem quadrupole mass spectrometer. Spectral analysis and biomass calculations were carried out on a DEC Station 5000 (Digital Equipment Corp.) using CHRO, SPEC and PEPmatch software (Finnigan).

For LC-MS, the tryptic peptides were dissolved in 5\% (v/v) acetonitrile in $0.1 \%$ trifluoroacetic acid and applied to a Vydac C18 reversed-phase column $(150 \times 0.8 \mathrm{~mm})$, directly coupled to the mass spectrometer. Peptides were eluted by a linear gradient from 5 to $95 \%$ acetonitrile in $0.1 \%$ trifluoroacetic acid and a flow rate of $20 \mu \mathrm{l} / \mathrm{min}$ provided by a dual-piston Pump 140B (Applied Biosystems).

For MS-MS the electrospray source head was replaced by an in-house built nanoelectrospray source. Nanospray needles and the Poros R2 desalting columns were self-made by pulling borosilicate glass capillaries (GC120F-10, Harvard Apparatus, Kent, UK) with a micropipette puller (Model P-87 Puller, Sutter Instrument Co., Novato, USA). Nanospray needles were gold coated in batches of 20 needles. For each sample a new desalting column and nanospray needle was used. Nano ES measurements were performed with about 600 to $1500 \mathrm{~V}$ applied to the spraying needle. For MS/MS mode 2-3 mtorr Argon was used in the collision cell and the collision energy was set to $10-15 \mathrm{eV}$ for daughter and to $35-50 \mathrm{eV}$ for parent ion scans. Q1 resolution was set to 2 units and Q3 was set to 1unit resolution. Plugged needles could be reopened by tipping the needle to the front side of the heated capillary with the needle at $200 \mathrm{~V}$ and the capillary at $40 \mathrm{~V}$ according to (Wilm and Mann, 1996).

Four pooled and lyophilised HPLC Peaks of the tryptic digest were dissolved in $10 \mu \mathrm{l} 5 \%$ acetonitrile/0.1\% trifluoroacetic acid and concentrated and desalted using $\sim 2 \mu$ of Poros R2 material (PerSeptive Biosystems, Cambridge, USA) placed in the tip pulled glass capillary similar to the description in (Wilm and Mann, 1996). The Poros R2 material was equilibrated with $5 \%$ acetonitrile $/ 0.1 \%$ trifluoroacetic acid and the bound peptides where washed with distilled water. Subsequently, the peptides where directly eluted into a nanospray needle with $5 \mu \mathrm{l} 80 \%$ acetonitrile $/ 5 \%$ formic acid.

\section{Visualisation and Quantification of the Hydrolysis or Phosphorylation}

Phosphorylation or GTP binding and hydrolysis of Toc34 $\Delta$ TM or outer envelope Toc34 by radioactive labelled nucleotides was visualised or quantified by different methods. For visualisation of radioactive probes, the gel or the DC-plate was exposed to a XOMAT-LS film (Kodak, Rochester, USA) or to a Phospho-Image plate (Fuji-Film, Tokyo, Japan). XTP hydrolysis was visualised by ultra violet light exposure $(254 \mathrm{~nm})$ and picturing using a digital camera Coolpix 880 (Nikon, Tokyo, Japan). Data were transferred to Adope Photoshop 5.0 LE. For quantification, the dried SDS-PAGE gel slices were dissolved in $30 \% \mathrm{H}_{2} \mathrm{O}_{2}$ and $60 \%$ $\mathrm{HClO}_{4}$ for $16 \mathrm{~h}$ at $60^{\circ} \mathrm{C}$ followed by scintillation counting. Alternatively, the Phospho-Image plate was scanned using a Phospho-Image Reader FLA 5000 (Fuji-Film, Tokyo, Japan) and quantified using Aida-Image Analyser (Raytest Isotopenmessgeräte $\mathrm{GmbH}$, Staubenhard, Germany). Data were presented by using Sigma Plot 5.0 (SPSS Inc., Chicago, USA).

\section{Acknowledgements}

We are grateful to Soumya Qbadou for the overexpressed Toc34 $\mathrm{C}_{\text {inv }}$ and Toc34 $\Delta_{2-119} \mathrm{C}_{\text {inv }}$ and to Michael Gutensohn for the DNA encoding for Arabidopsis thaliana Toc33 $\Delta \mathrm{TM}$. This work was supported by grants from the Deutsche Forschungsgemeinschaft and the Human Frontier Science Programm.

\section{References}

Albert, S., Will, E. and Galwitz, D. (1999). Identification of the catalytic domains and their functionally critical arginine residues of two yeast GTPase-activating proteins specific for Ypt/Rab transport GTPases. EMBO J. 18, 5216-5225.

Bauer, J., Chen, K., Hiltbunner, A., Wherli, E., Eugster, M., Schnell, D. and Kessler, F. (2000). The major protein import receptor of plastids is essential for chloroplast biogenesis. Nature 403, 203-207.

Bölter, B., May, T. and Soll, J. (1998). A protein import receptor in pea chloroplasts, Toc86, is only a proteolytic fragment of a larger polypetide. FEBS Lett. 441, 59-62.

Bourne, H. R., Sanders, D. A. and McCormick, F. (1991). The GTPase superfamily: conserved structure and molecular mechanism. Nature 349, 117-127.

Caliebe, A., Grimm, R., Kaiser, G., Lübeck, J., Soll, J. and Heins, L. (1997). The chloroplastic protein import machinery contains a Rieske-type iron-sulfur cluster and a mononuclear ironbinding protein. EMBO J. 16, 7342 - 7350.

Cherfils, J. and Chardin, P. (1999). GEFs: structural basis for their activation of small GTP-binding proteins. Trends Biol. Sci. 24, $306-311$.

Deltrot, S., Atanassova, R. and Maurousset, L. (2000). Regulation of sugar, amino acid and peptide plant membrane transporters. Biochim. Biophys. Acta 1465, 281-306.

Downward, J., Riehl, R., Wu, L. and Weinberg, R. A. (1990). Identification of a nucleotide exchange promoting activity for p21ras. Proc. Natl. Acad. Sci. USA 87, 5998-6002.

Flügge, U. I. and Hinz, G. (1986). Energy dependence of protein translocation into chloroplasts. Eur. J. Biochem. 160, 563570.

Gruss, O., Feick, P., Frank, R. and Dobberstein, B. (1999). Phosphorylation of components of the ER translocation site. Eur. J. Biochem. 260, 785-793.

Gutensohn, M., Schulz, B., Nicolay, P. and Flügge, U.-I. (2000). Functional analysis of the two Arabidopsis homologues of Toc34, a component of the chloroplast protein import apparatus. Plant J. 23, $771-783$.

Hardie, D. G. (1999). Plant protein serine/threonine kinases: classification and functions. Annu. Rev. Plant Physiol. Plant Mol. Biol. 50, 97-131.

Hiltbrunner, A., Bauer, J., Vidi, P. A., Infanger, S., Weibel, P., Hohwy, M. and Kessler, F. (2001). Targeting of an abundant cytosolic form of the protein import receptor atToc159 to the outer chloroplast membrane. J. Cell Biol. 154, 309-316.

Hirsch, S., Muckel, E., Heemeyer, F., von Heijne, G. and Soll, J. (1994). A receptor component of the chloroplast protein translocation machinery. Science 266, 1989-1992.

Hwang, Y. W. and Miller, D. L. (1987). A mutation that alters the nucleotide specificity of elongation factor Tu, a GTP regulatory protein. J. Biol. Chem. 262, 13081-13085.

Keegstra, K. and Cline, K. (1999). Protein import and routing systems of chloroplasts. Plant Cell 11, 557-570.

Kessler, F., Blobel, G., Patel, H. A. and Schnell, D. J. (1994). Identification of two GTP-binding proteins in the chloroplast protein import machinery. Science 266, 1035-1039. 
Kouranov, A. and Schnell, D. J. (1997). Analysis of the interactions of preproteins with the import machinery over the course of protein import into chloroplasts. J. Cell Biol. 139, $1677-$ 1685.

May, T. and Soll, J. (1998). Positive charges determine the tropology and functionality of the transmembrane domain in the chloroplastic outer envelope protein Toc34. J. Cell Biol. $141,895-904$.

Olsen, L. J. and Keegstra, K. (1992). The binding of precursor proteins to chloroplasts requires nucleoside triphosphates in the intermembrane space. J. Biol. Chem. 267, 433- 439.

Olsen, L. J., Theg, S. M., Selman, B. R. and Keegstra, K. (1989). ATP is requierd for the binding of precursor proteins to chloroplasts. J. Biol. Chem. 264, 6724-6729.

Pain, N. (1996). Initiation of protein synthesis in eukaryotic cells. Eur. J. Biochem 236, 747-771.

Robinson, P. J., Sontag, J. M., Liu, J. P., Fykse, E. M., Slaughter, C., McMahon, H. and Sudhof, T. C. (1993). Dynamin GTPase regulated by protein kinase $\mathrm{C}$ phosphorylation in nerve terminal. Nature 365, 163-166.

Ruetthard, H., Banerjee, A. and Makinen, M. W. (2001). $\mathrm{Mg}^{2+}$ is not catalytically required in the intrinsic and kirromycin-stimulatted GTPase action of Thermus thermophilus EF-Tu. J. Biol. Chem. 276, 18728-18733.

Scheffzek, K., Ahmadian, M. R. and Wittinghofer, A. (1998). GTPase-activating proteins: helping hands to complement an active site. Trends Biol. Sci. 23, 257-262.

Schindler, C., Hracky, R. and Soll, J. (1987). Protein transport in chloroplasts: ATP is prerequisite. Z. Naturforsch. 42c, 103108.

Schleiff, E. and Soll, J. (2000). Travelling of proteins through membranes: translocation into chloroplasts. Planta 211, 449-456.

Schleiff, E., Soll, J., Sveshnikova, N., Tien, R., Wright, S., Dabney-Smith, C., Subramanian, C. and Bruce, B. D. (2002). Structural and GTP/GDP requirements for transit peptide recognition by the cytosolic domain of the chloroplast outer envelope receptor, Toc34. Biochemistry 41,1934-1946.

Schmidt, G., Lenzen, C., Simon, I., Deuter, R., Cool, R. H., Goody, R. S. and Wittinghofer, A. (1996). Biochemical and bi- ological consequences of changing the specificity of p21ras from guanosine to xanthosine nucleotides. Oncogene 12, 87-96.

Schnell, D., Blobel, G., Keegstra, K., Kessler, F., Ko, K. and Schnell, J. (1997). A consensus nomenclature for the proteinimport components of the chloroplast envelope. Trends Cell Biol. 7, 303-304.

Seedorf, M., Waegemann, K. and Soll, J. (1995). A constituent of the chloroplast import complex represents a new type of GTPbinding protein. Plant J. 7, 401-411.

Soll, J., Fischer, I. and Keegstra, K. (1988). A guanosine 5'triphosphate-dependent protein kinase is localized in the outer envelope of pea chloroplast. Planta 176, 488-496.

Su, Q., Schild, C., Schumann, P. and Boschetti, A. (2001). Effect of precursor protein phosphorylation on import into isolated chloroplasts from Chlamydomonas. Eur. J. Biochem. 268, 2315-2321.

Sun, Y.-J., Forouhar, F., Li, H.-m., Tu, S.-L., Yeh, Y.-H., Kao, S., Shr, H.-L., Chou, C.-C., Chen, C. and Hsiao, C.-D. (2002). Crystal structure of pea Toc34, a novel GTPase of the chloroplast protein translocon. Nature Struct. Biol. 9, 95-100.

Sveshnikova, N., Soll, J. and Schleiff, E. (2000). Toc34 is a preprotein receptor regulated by GTP and phosphorylation. Proc. Natl. Acad. Sci. USA 97, 4973-4978.

Waegemann, K. and Soll, J. (1996). Phosphorylation of the transit sequence of chloroplast precursor proteins. J. Biol. Chem. 271, 6545-6554.

Wilm, M. and Mann, M. (1996). Analytical properties of the nanoelectrospray ion source. Anal. Chem. 68, 1-8.

Young, M. E., Keegstra, K. and Froehlich, J. E. (1999). GTP promotes the formation of early-import intermediates but is not required during the translocation step of protein import into chloroplast. Plant Phys. 121, 237-243.

Zhang, B., Wang, Z. X. and Zheng, Y. (1997). Characterisation of the interactions between the small GTPase Cdc42 and its GTPase activating proteins and putative effectors. J. Biol. Chem. 272, 21999-22007.

Received May 14, 2002; accepted July 17, 2002 